\title{
Real-Time TEM Imaging of Moisture-Induced Degradation of Triple Cation Mixed Halide Perovskite
}

$\underline{\text { Nicolas Folastre }^{1}}$, Mohammad Ali Akhavan Kazemi $^{1}$, Arash Jamali ${ }^{1}$, Frédéric Sauvage ${ }^{1}$ and Arnaud Demortiere $^{1 *}$

${ }^{1}$ Laboratoire de Réactivité et Chimie des Solides (LRCS), UMR CNRS 7314-UPJV, Amiens, France.

The actual growth of the global energy demand in the context of the energetic transition calls an augmentation of the energy production based on green sources as low-cost photovoltaics (PVs). In the last few years, the perovskite solar cells (PSCs) technology reached a first plan place among PVs. The unprecedented growth in the power conversion efficiency (PCE) of PSCs from 3.8\% in 2009 to $25.5 \%$ highlighted this technology as a promising candidate to replace the conventional Si-based and thin-film solar cell technologies [1]. One of the major challenges in manufacturing low-cost, high-performance tandem devices was the lack of a high-efficiency upper cell with a band gap greater than $1.5 \mathrm{~V}$. The recently introduced organic / inorganic hybrid perovskite solar cells allow to overcome these limitations due to their unique advantages. Recently, one of the best performance among mixed organic-inorganic halide perovskite (HPs) came with the triple cations/double halides $\mathrm{Cs}_{0.05}\left(\mathrm{MA}_{0.17} \mathrm{FA}_{0.83}\right)_{0.95} \mathrm{~Pb}\left(\mathrm{Br}_{0.17} \mathrm{I}_{0.83}\right)_{3}$ (CsMAFA) composition in single junction cell [2], that also appeared as one of a top cell in a monolithic perovskite/silicon tandem architecture reaching above $29 \%$ PCE [3]. Despite promising PCEs, the main obstacle for the future commercialization of perovskite-based solar cells is their lack in stability when exposed moisture for extended periods. Several ex-situ studies have emerged providing clues to determine the weaknesses of the materials [4].Recently, the development of TEM liquid cells [5] provided a large panel of techniques to characterize at micro- and nano-scale the solid-liquid interfaces, allowing dynamical observation of crystallization processes of nanomaterials in solutions with high resolution and fast acquisitions [6]. In this work, for the very first time, we successfully monitored by in situ liquid-cell TEM imaging the moisture-induced phase segregation of CsMAFA (Fig. 1), also characterised by EDX, STEM, SAED and 4D-STEM-ASTAR [7]. The phase and orientation maps acquired in 4D-STEM added to the elementary EDX maps made it possible to determine the species formed after the segregation of the CsMAFA phases in water. The particles were characterized by STEM and TEM imaging, The growth kinetics of these grains were quantified after segmentation of the TEM images (Fig. 2) showing different growth rates according to various morphologies of grains. The particle growth is influenced by several factors described by the LSW as limitation by diffusion, but also by the density of the species present in the liquid which significantly affect the growth of neighboring grains. The different techniques used around the liquid TEM cell have made it possible to follow the different stages of degradation of CsMAFA in water from the pristine state, through phase segregation and finally partial dissolution in water. The results presented here provide answers around the resolution of humidity instability problems of hybrid perovskite devices.References:[1] M.A. Green et al, The emergence of perovskite solar cells, Nature Photon. [2] J.-P. Correa-Baena et al, Promises and challenges of perovskite solar cells, Science. 358 (2017)

References:

[1] MA Green et al, Nature Photonics 8 (2014), p. 506.

[2] J-P Correa-Baena et al, Science 358 (2017).

[3] A Al-Ashouri et al, Science 370 (2020), p. 1300.

[4] TA Berhe et al, Energy Environ. Sci. 9 (2016), p. 323. 
[5] Protochips, Micros. Today. 25 (2017), p. 13.

[6] OM Karakulina et al, Nano Lett. 18 (2018), p. 6286.

[7] EF Rauch et al, SSP 186 (2012), p. 13.
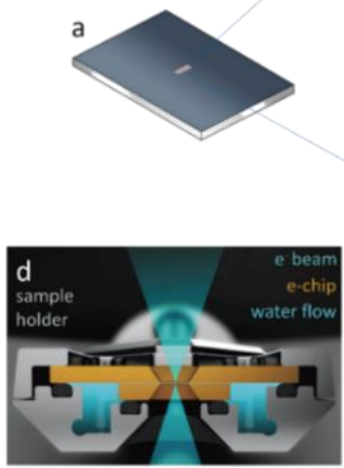
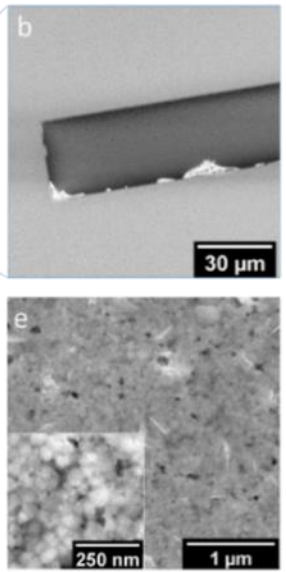
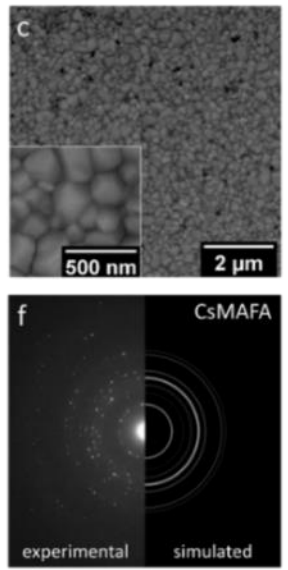

Figure 1. (a) TEM e-chip of the Protochip Poseidon Select liquid cell[10] and (b, c) the scanning electron microscopy (SEM) images of the CsMAFA sample deposited by spin-coating on the e-chip window. The size of the grains goes up to $500 \mathrm{~nm}$. The distribution of the particle size is relatively homogeneous in the pristine. (d) Scheme of Protochip Poseidon Select liquid cell composed of two chips separated by a spacer and sealed by a gasket. The gap between the chips allows liquid to pass in between in contact with the sample, the windows of the chip allow the e- beam to pass through both the sample and the fluid. (e) Scanning transmission electron microscopy (STEM) of the pristine sample under a $200 \mathrm{kV}$ electron beam (f) Selected area electron diffraction (SAED) (left) compared to simulation with MacTempasX software of CsMAFA pristine sample (right).

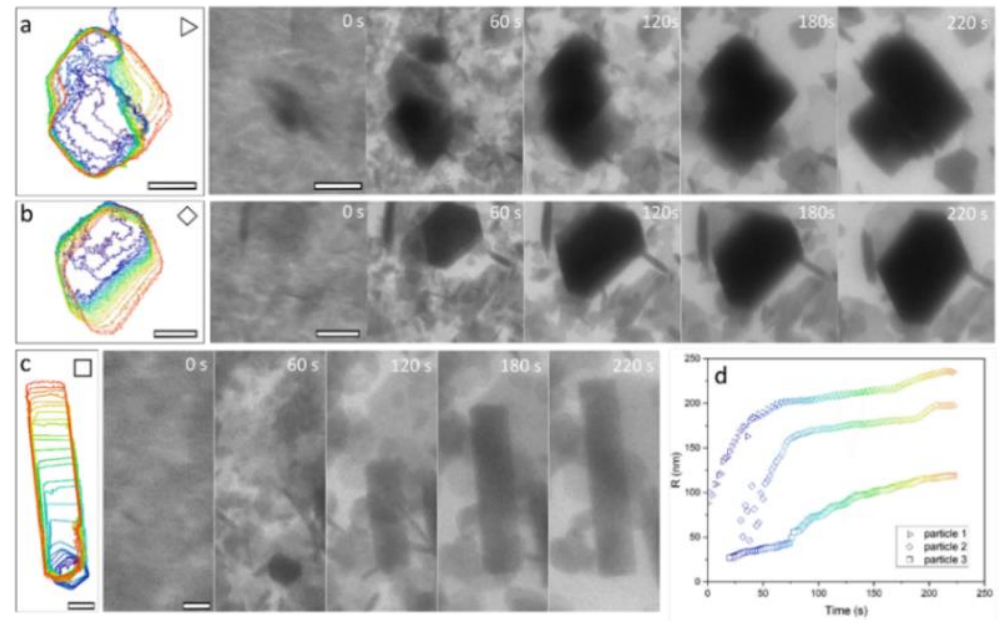

Figure 2. $(a, b, c)$ Growth of well-shaped particles in water tracked by TEM. The particles grow in water and reach different sizes up to about $500 \mathrm{~nm}$ depending on their largest dimension. The corresponding TEM images were segmented with WEKA segmentation 3D plugin in ImageJ. Each color refers to a specific time. All scale bars are $200 \mathrm{~nm}$. (d) The evolution of the normalized radius of the particles by time of water contact. The radius is normalized as the radius of a disk of same surface than the particle. The TEM images are summed and normalized along the $\mathrm{Z}$ axis of the image stack to increase the $\mathrm{SNr}$. The color code is the same on the segmented images and on the graph. 